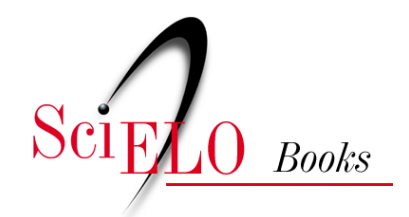

EdUfSCar

\title{
7 - Simulação por dinâmica molecular da enzima cruzaína do trypanosoma cruzi
}

\author{
Osmair Vital de Oliveira
}

\section{SciELO Books / SciELO Livros / SciELO Libros}

OLIVEIRA, O.V. Simulação por dinâmica molecular da enzima cruzaína do trypanosoma cruzi. In: FREITAS, L.C.G., and OLIVEIRA, G.S., orgs. Aplicações de química teórica no estudo de materiais: métodos in silico para nanomateriais [online]. São Carlos: EdUFSCar, 2018, pp. 227-244. ISBN 97865-80216-12-3. Avalaible from: doi: 10.7476/9786580216123.0008. Also available in ePUB from: http://books.scielo.org/id/nvnjd/epub/freitas-9786580216123.epub.

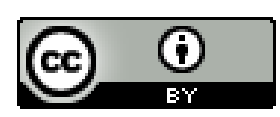

All the contents of this work, except where otherwise noted, is licensed under a Creative Commons Attribution $\underline{4.0 \text { International license. }}$

Todo o conteúdo deste trabalho, exceto quando houver ressalva, é publicado sob a licença Creative Commons Atribição 4.0.

Todo el contenido de esta obra, excepto donde se indique lo contrario, está bajo licencia de la licencia Creative Commons Reconocimento 4.0. 


\section{SIMULAÇÃO POR DINÂMICA MOLECULAR DA ENZIMA CRUZAÍNA DO TRYPANOSOMA CRUZI}

Osmar Vital de Olveira?

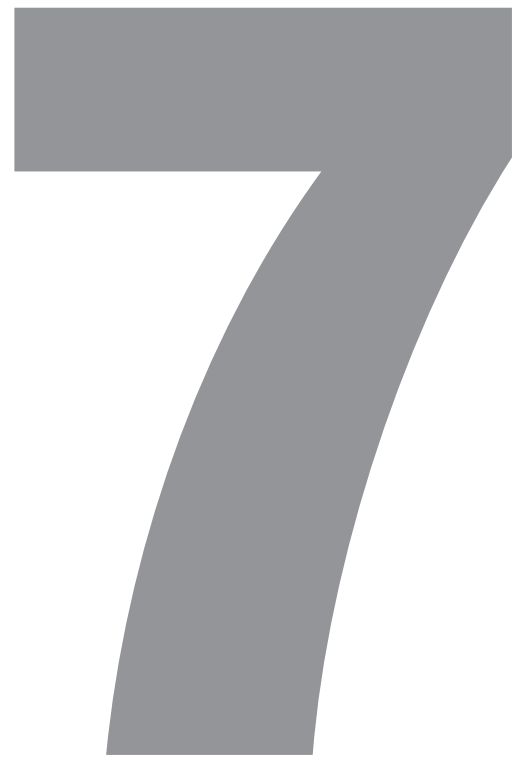




\subsection{INTRODUÇão}

As enzimas são de fundamental importância para o funcionamento e a sobrevivência dos seres vivos. Portanto, compreender as relações entre estrutura e função de enzimas é fundamental para entender a intrincada teia dos processos vitais. Entre os vários processos vitais, estudar o mecanismo das reações enzimáticas é uma tarefa importante e decisiva para a compreensão dos mecanismos moleculares em sistemas biológicos. Em reações enzimáticas, esses estudos implicam conhecer o entendimento das interações entre o substrato e o sítio catalítico da enzima, bem como de outros fatores que influem nessa interação. Dentre esses fatores, destacam-se a composição e estrutura do sítio ativo e efeitos dinâmicos do sistema. Ressalta-se que entender a sinergia desses processos é de real importância para o desenvolvimento de estratégias de intervenção em processos patológicos. Entre as intervenções, cabe destacar o desenvolvimento de novos fármacos.

Para entender melhor os processos enzimáticos, é necessário tratar o sistema nos níveis atômico e molecular. Entretanto, são raras as técnicas experimentais que revelam detalhes completos da dinâmica desses sistemas em escala atômica. As poucas técnicas existentes possuem custo elevado e dificuldades práticas para a aplicação generalizada, sendo, portanto, pouco acessíveis à totalidade da comunidade científica. Desse modo, com o avanço e a popularização de computadores cada vez mais velozes e de baixo custo, a química computacional consolida-se gradativamente como uma área de pesquisa fundamental para o entendimento de processos químicos nos níveis atômico e molecular. A sinergia existente entre o desenvolvimento de hardware e software cria novos horizontes para a aplicação em pesquisa científica. Os avanços obtidos têm sido de grande valia para os químicos teóricos, proporcionando também o surgimento de interfaces de colaboração direta com a pesquisa experimental.

Dentre as diversas metodologias de química teórica utilizadas para estudar sistemas biológicos, a dinâmica molecular é uma técnica muito interessante, pois 
utiliza campos de força que são muito úteis para a obtenção de propriedades termodinâmicas e estruturais de líquidos e macromoléculas. Ademais, a eficiência dessa metodologia permite que um número relativamente grande de partículas seja convenientemente tratado. Assim, a técnica dinâmica molecular será utilizada para estudar a enzima cisteína protease cruzaína do Trypanosoma cruzi, responsável pela doença de Chagas. Alguns objetivos são: i) estudar a enzima cruzaína; ii) caracterizar o comportamento dinâmico dessa enzima na presença e ausência de uma cadeia peptídica; iii) utilizar esse conjunto de informações para identificar as interações que podem contribuir para o desenho racional de fármacos; e iv) elaborar conjunto de ancoragem, a fim de ser utilizado em futuros trabalhos para a proposição de novos inibidores eficientes.

\subsection{Doença de Chagas}

A doença de Chagas foi descoberta em 1909 pelo médico sanitarista Carlos Chagas, causada pelo protozoário Trypanosoma cruzi, que é transmitido principalmente pelo inseto-vetor hematófago conhecido popularmente como barbeiro. Esse inseto é classificado na ordem Hemiptera e família Reduviidae, sendo encontrado com frequência em frestas de paredes de barro ou de madeira de lares na zona rural e em outras localidades. Uma das maneiras mais eficientes que tem sido utilizada no controle da doença de Chagas é a eliminação de ambientes favoráveis à procriação do inseto-vetor, bem como a eliminação deste por meio de inseticidas. Outras formas de transmissão da doença ocorrem por transfusão de sangue e transmissão congênita. ${ }^{2} \mathrm{~A}$ figura a seguir apresenta as áreas de maiores incidências e de riscos da doença de Chagas.

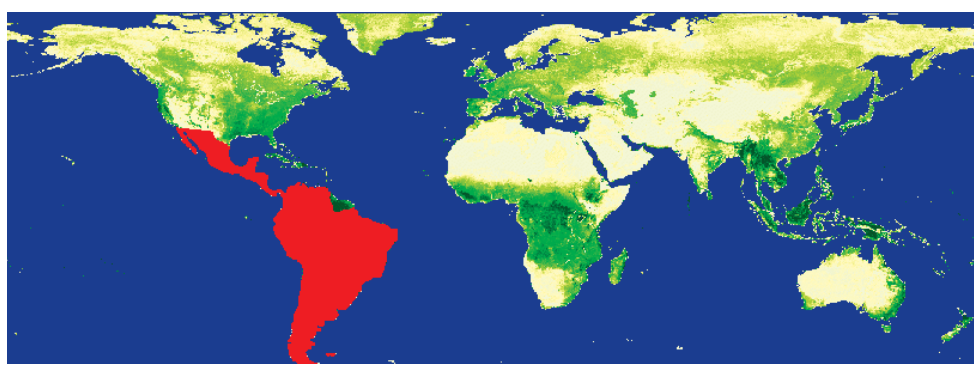

Figura 7.1 Distribuição global (em vermelho) da doença de Chagas.

Fonte: <http://geo.arc.nasa.gov/>.

Estima-se que a cada ano 200 mil pessoas são contaminadas pela doença de Chagas e 40 milhões de pessoas se encontram com risco de contrair a doença. Desse

2 WHO (2002). 
total, cerca de 13 mil pessoas morrem em decorrência de complicações pela doença. ${ }^{3}$ A doença de Chagas envolve dois estágios clínicos: agudo e crônico. ${ }^{4} \mathrm{Na}$ fase aguda, o estágio se inicia após um inseto infectado picar uma pessoa. Alguns sintomas nessa fase são inchaço no local da picada, febre e taquicardia. A fase de infecção aguda normalmente não é fatal. Já na fase crônica, o estágio é dividido em dois, sintomático e assintomático. Cerca de $60 \%$ dos pacientes chagásicos crônicos permanecem assintomáticos por longos períodos, sendo que $30 \%$ deles desenvolvem complicações cardíacas que podem levar à cardiopatia chagásica crônica.

Apesar de bem conhecida há mais de cem anos, até os dias atuais existem apenas dois fármacos contra a doença: benzonidazol e nifurtimox. Entretanto, apresentam baixa eficiência e fortes efeitos colaterais, ${ }^{5}$ bem como atividade inibitória apenas na fase aguda da doença. No Brasil, a comercialização do composto nifurtimox é proibida. ${ }^{6} \mathrm{O}$ baixo desenvolvimento de fármacos antichagásicos pode ser atribuído ao pouco interesse da indústria farmacêutica, o que se deve principalmente à falta de incentivos comerciais, ou seja, baixa demanda.

Para o desenvolvimento de fármacos contra o parasita, vários processos bioquímicos têm sido apontados e explorados como alvos terapêuticos potenciais: ${ }^{7}$ a enzima tripanotiona redutase; ${ }^{8}$ enzimas envolvidas na importação e interconversão de purinas; $;$ metabolismo de folato e pteridina; ${ }^{10}$ biossíntese de RNA mensageiro; ${ }^{11}$ biossíntese de lipídios; transialidase, ${ }^{12}$ diidrofolato redutase, ${ }^{13}$ enzimas do glicossomo, envolvidas no metabolismo energético, destacando-se a gliceraldeído-3-fosfato desidrogenase, ${ }^{14}$ e outros.

Outro alvo terapêutico que tem sido extensivamente explorado são as enzimas proteases de cisteína, em especial a cruzaína (EC 3.4.22.51). Proteases são enzimas que catalisam a hidrólise de ligações peptídicas. As proteases podem ser divididas em sete classes, de acordo com seu mecanismo catalítico. Segundo esta classificação, os membros de cada uma destas classes possuem um conjunto característico de aminoácidos funcionais dispostos em uma configuração específica e que formam o centro ativo da enzima. As diferentes classes se subdividem, ainda, em diversas famílias, sendo que os membros de famílias diferentes, apesar de terem uma sequência de aminoácidos distinta, possuem geometria e mecanismo catalítico semelhantes, ${ }^{15}$ em que cada peptidase tem função específica no organismo humano

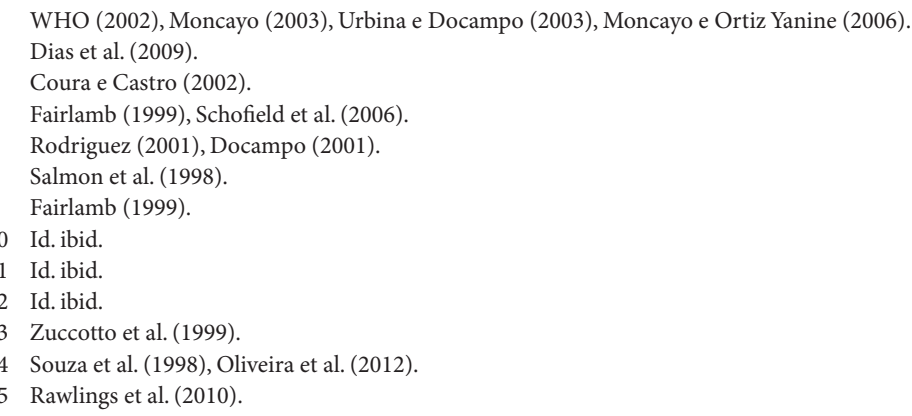


e corresponde de $1 \%$ a $5 \%$ de seus conteúdos genéticos. Dentre as proteases, têm-se as cisteínas proteases, as quais possuem uma cisteína no sítio ativo. Enzimas dessa família têm diversas funções no organismo, como, por exemplo, serem essenciais na apoptose celular e necessárias no sistema imune. ${ }^{16}$ Desse modo, disfunções dessas enzimas podem levar a vários processos fisiopatológicos, como o desenvolvimento de tumores e doenças autoimunes.

É bom enfatizar que enzimas dessa família estão presentes em diversos parasitas, como, por exemplo, Trypanosoma cruzi e Leishmania, e desempenham neles funções vitais. ${ }^{17}$ Portanto, enzimas proteases de cisteína são alvos terapêuticos interessantes no desenvolvimento de fármacos, uma vez que sua inibição pode levar à cura de doenças como câncer e doença de Chagas. A cruzaína pertencente à superfamília papaína é a protease mais abundante e melhor caracterizada do parasita $T$. cruzi. ${ }^{18}$ Essa enzima é necessária e essencial para a sobrevivência do parasita, para sua diferenciação celular e seu crescimento e desenvolvimento dentro da célula hospedeira. ${ }^{19}$ Além disso, a cruzaína participa do processo de invasão e internalização do parasita nas células de mamíferos. O mecanismo de hidrólise de peptídeos da cruzaína é o mesmo das cisteíno-proteases, pois o sítio catalítico é constituído pelos aminoácidos cisteína, histidina e glutamina.

A função da glutamina no processo catalítico é estabilizar o par iônico cisteína/ histidina. É bom enfatizar que, desde 2001, a cruzaína é um alvo validado para a quimioterapia da doença de Chagas. ${ }^{20}$ Assim, a inibição dessa enzima pode levar à morte do parasita. Com este objetivo, vários estudos têm sido mencionados na literatura, tanto no nível teórico quanto no experimental. Experimentalmente, derivados peptídicos ${ }^{21}$ e não peptídicos (triazóis, pirimidinas, tiossemicarbazonas e chalconas) ${ }^{22}$ têm sido estudados na tentativa de obter um fármaco para inibir a cruzaína. Já nos estudos teóricos, podem ser citados os cálculos de dinâmica molecular, ${ }^{23}$ ancoragem e triagem virtual..$^{24}$ Apesar de todos esses esforços, até a presente data, ainda não foi descoberto um composto eficiente para a inibição da cruzaína do T. cruzi.

Portanto, visando entender o comportamento estrutural e energético e as interações específicas da enzima cruzaína na forma apo (não complexada) e na forma holo (complexada com um peptídeo), foram realizadas simulações por dinâmica molecular. A Figura 7.2 a seguir mostra a estrutura cristalográfica da cruzaína obtida no Protein Data Bank.

6 Leung et al. (2000).

17 Turk (2006).

18 Cazzulo et al. $(1997,2001)$

19 Cazzulo et al. (2001), Reis et al. (2006), McKerrow et al. (2006).

20 Cazzulo et al. (2001).

21 Andricopulo et al. (1998), McKerrow et al. (2008).

22 Zanatta et al. (2008), Soeiro e Castro (2009), Chen et al. (2010).

23 Mladenovic et al. (2008), Durrant et al. (2010).

24 Malvezzi et al. (2009), Romeiro et al. (2009), Durrant et al. (2010). 


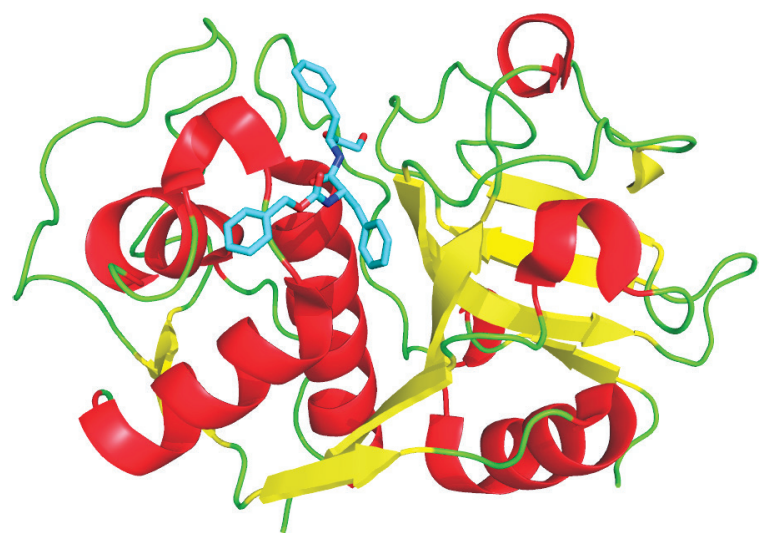

Figura 7.2 Estrutura cristalográfica da cruzaína complexada com um inibidor covalente (código PDB: 1ME4).

Fonte: $<$ www.pdb.org/>.

\subsection{Protocolo de dinÂMICA MOLECULAR}

Dinâmica molecular (DM) é uma metodologia utilizada para resolver problemas em mecânica estatística, na impossibilidade de solução analítica das equações. Em sua forma geral, a DM é utilizada para simular a evolução temporal de um sistema sob a ação de forças conhecidas. Essa metodologia recorre à integração das equações de movimento clássicas para determinar aceleração, velocidade e posição temporal das partículas do sistema. ${ }^{25} \mathrm{~A}$ partir da trajetória gerada, várias propriedades podem ser determinadas: coeficiente de difusão, energia de interação, parâmetros estruturais, entre outras.

Para a construção dos sistemas a serem simulados, a estrutura cristalográfica da enzima cruzaína foi obtida no Protein Data Bank, com código PDB 1ME4. Para a simulação da proteína na forma apo, o ligante cristalográfico foi removido, e a enzima foi inserida em uma caixa cúbica de aresta medindo $8 \mathrm{~nm}$, contendo 16.091 moléculas de água. Para manter o sistema eletrostaticamente neutro, 13 íons $\mathrm{Na}^{+}$ foram adicionados pela replicação de moléculas de água preexistentes. Para a simulação do sistema na forma holo, foi necessário obter a estrutura do peptídeo de outra cisteína protease, uma vez que, até a presente data, ainda não foi cristalizada a cruzaína com alguma cadeia peptídica. Assim, a catepsina K humana complexada com um polipeptídeo de 99 aminoácidos foi obtida no Protein Data Bank (código PDB 1BY8) e sobreposta com a estrutura da cruzaína. A partir dessa sobreposição, as coordenadas cristalográficas do polipeptídeo (Lys-Met-Thr-Gly-Leu-Lys-Val-Pro) foram inseridas dentro da estrutura 1ME4 (cruzaína). Esta sequência de aminoáci- 
dos (que forma um loop) foi considerada, pois apenas estes ocupam a região do sítio ativo da enzima. A Figura 7.3 a seguir mostra o complexo cruzaína-peptídeo obtido.

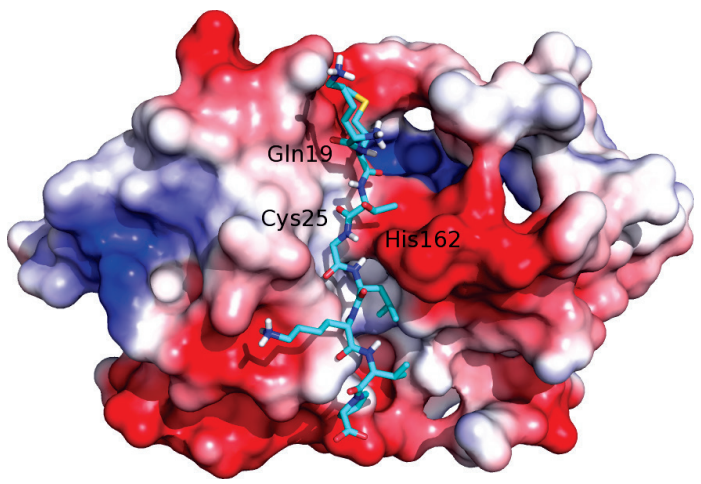

Figura 7.3 Complexo cruzaína-peptídeo a ser simulado. A cruzaína é representada por seu potencial eletrostático (negativo em vermelho, positivo em azul e cargas neutras em branco), e a posição da tríade catalítica é mostrada em detalhe. O peptídeo está em stick, e, para melhor visualização, os hidrogênios apolares foram removidos.

O complexo cruzaína-peptídeo foi solvatado com 16.081 moléculas de água e foram adicionados 11 íons $\mathrm{Na}^{+}$para manter a neutralidade do sistema. Condições periódicas de contorno foram adicionadas, e um raio de corte de $1,0 \mathrm{~nm}$ foi utilizado para as interações de Coulomb e Van der Waals. O conjunto NpT (número de átomos, pressão e temperatura constantes) foi utilizado. A pressão e a temperatura do sistema adotadas foram de 1 bar e $310 \mathrm{~K}$, respectivamente, com constantes de tempo de 1,0 ps e $0,1 \mathrm{ps}$, respectivamente, utilizadas no algoritmo termostato e barostato de Berendsen. ${ }^{26}$ Para descrever o solvente, foi utilizado o modelo SPC/ flexível ${ }^{27}$; para a enzima, foram considerados parâmetros do campo de força OPLS- $\mathrm{AA}^{28}$. Para minimizar o efeito de contato ou da sobreposição de átomos entre as moléculas, foi realizada a minimização da energia do sistema com os algoritmos máximo decline e gradiente conjugado. Nessas etapas, mil passos de minimização foram utilizados em ambos algoritmos. Após essa etapa, uma trajetória de 500 ps foi gerada, restringindo os movimentos internos na enzima com constantes de força de $1.000 \mathrm{~kJ} \cdot \mathrm{mol}^{-1} \cdot \mathrm{nm}^{-2}$. O objetivo dessa etapa foi relaxar e termalizar o solvente na temperatura desejada. Finalizada essa etapa, gerou-se uma trajetória de 50,0 ns de simulação, e o mesmo procedimento foi feito para a enzima na forma apo, mas, nesse caso, apenas removeu-se a cadeia peptídica da região do sítio ativo. Dentre os vários pacotes computacionais de dinâmica molecular, aqui foi utilizado o Gromacs. ${ }^{29}$

26 Berendsen et al. (1984).

27 Id. (1981).

28 Jorgensen et al. (1996).

29 Berendsen et al. (1995), Lindahl et al. (2001). 


\subsection{ANÁLISES DE DINÂMICA MOLECULAR}

Aqui, apresentar-se-ão algumas principais análises e discussões que são realizadas em sistemas biológicos estudados por dinâmica molecular com enfoque na obtenção de fármacos.

\subsubsection{Análise estrutural}

Uma medida inicial e importante em simulação por DM de enzimas é o cálculo do desvio quadrático médio ou RMSD (Root Mean Square Deviation). O RMSD fornece a medida do desvio da estrutura de uma dada molécula ao longo do tempo. Para calculá-lo, a cada intervalo de tempo, executa-se uma sobreposição da estrutura atual com a inicial para obter o desvio entre ambas, o qual é mostrado na Equação 7.1.

$$
R M S D\left(t_{i}, t_{0}\right)=\left[\frac{1}{N} \sum_{i=1}^{N}\left\|r_{i}\left(t_{i}\right)-r_{0}\left(t_{0}\right)\right\|^{2}\right]^{\frac{1}{2}}
$$

em que $r_{\mathrm{i}}$ e $t_{\mathrm{i}}$ representam a posição do átomo $i$ no tempo; $t_{\mathrm{i}}$ e $t_{0}$ se referem à estrutura da molécula no tempo zero ou em um tempo predefinido. Logo, pequenas variações de valores de RMSD implicam baixa mudança conformacional ou baixa mobilidade. Embora experimentalmente não seja possível obter a evolução estrutural via RMSD, este pode ser correlacionado com uma propriedade experimental, o fator $\mathrm{B}$ de temperatura. $\mathrm{O}$ fator de temperatura é uma medida da posição atômica oriunda das vibrações térmicas de um sistema cristalino. A correlação entre RMSD e fator B de temperatura é expressa pela Equação 7.2.

$$
\mathrm{B}=(8 / 3) \mathrm{p}^{2}(\mathrm{RMSD})^{2}
$$

Portanto, usando a Equação 7.2 é possível correlacionar a variação estrutural (RMSD) obtida em uma trajetória de DM com uma observável experimental (fator B). Desse modo, para entender o comportamento estrutural da enzima cruzaína nas formas apo e holo, foram realizadas medidas de RMSD (Figura 7.4) e considerados todos os átomos $\mathrm{C} \alpha$. 


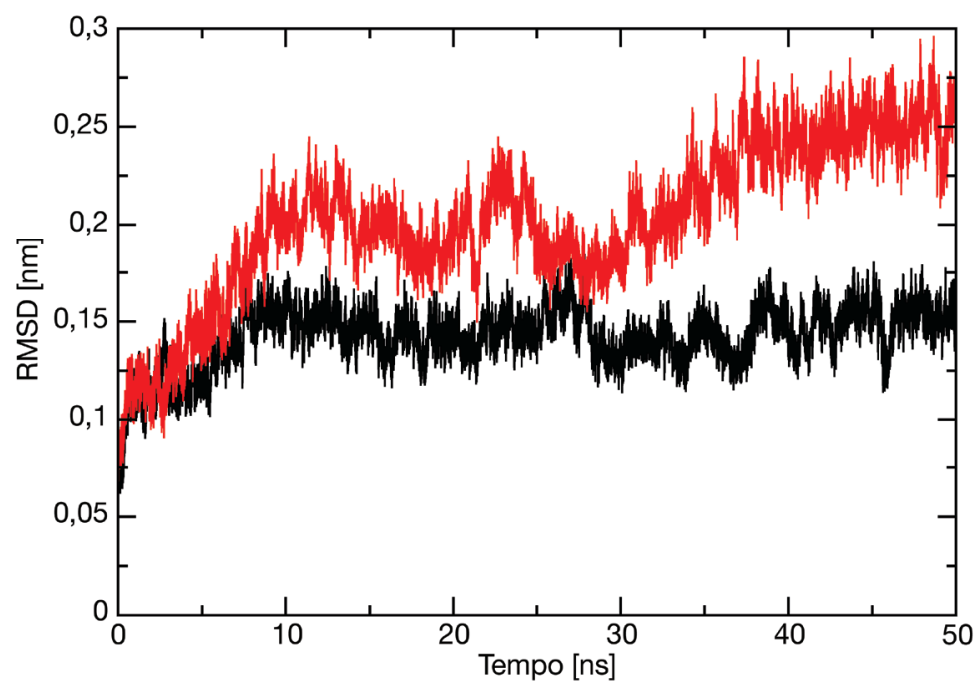

Figura 7.4 RMSD dos átomos C $\alpha$ da enzima cruzaína nas formas apo (linha vermelha) e holo (linha preta) obtidos ao longo da simulação.

A Figura 7.4 mostra que os valores de RMSD aumentam nos primeiros $10 \mathrm{~ns}$ de simulação de 0 para 0,15 e 0,20 $\mathrm{nm}$ para as formas holo e apo, respectivamente. Isso mostra que a enzima cruzaína perde sua conformação inicial. Depois desse tempo, o RMSD mantém média de $0,15 \mathrm{~nm}$ com desvio padrão (d.p.) de 0,01 nm, ao longo do tempo, para a forma holo. Já para a forma apo, o RMSD mantém média de $0,20 \mathrm{~nm}$, com d.p. de 0,02 nm no intervalo de 10 a $34 \mathrm{~ns}$. Depois desse tempo, o RMSD aumenta para $0,24 \mathrm{~nm}$, no tempo de $37 \mathrm{~ns}$, e, posteriormente, mantém média de $0,25 \mathrm{~nm}$, com d.p. de 0,01 nm. Essas análises mostram que a estrutura da enzima na forma apo passa por duas mudanças estruturais no tempo de simulação considerado, uma de 0 a 10 ns e outra de 34 a 37 ns de simulação. O baixo valor do RMSD na forma holo mostra que a estrutura da cruzaína não apresenta mudanças significativas ao longo da simulação. Ao contrário da forma holo, os valores relativamente altos de RMSD da forma apo mostram que a enzima apresenta mudanças conformacionais significativas. Esses dados revelam que a presença do peptídeo na região do sítio ativo da cruzaína estabiliza sua estrutura. Para entender quais regiões da enzima são responsáveis pelas mudanças observadas, foram realizadas análises de RMSF (Root Mean Standard Fluctuations). O RMSF mede a flutuação dos aminoácidos, ou parte deles, ao longo da simulação e, com isso, é possível identificar as principais regiões da enzima que apresentam maior flexibilidade. Na Figura 7.5, a seguir, tem-se o RMSF obtido na simulação para as formas apo e holo. 


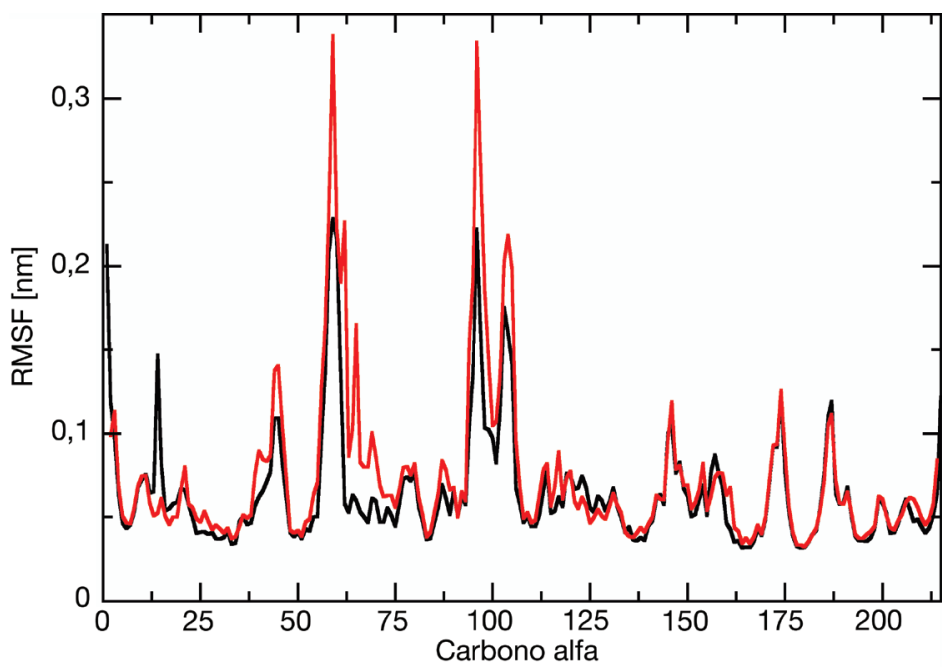

Figura 7.5 RMSF dos átomos C $\alpha$ da enzima cruzaína nas formas apo (linha vermelha) e holo (linha preta).

Em geral, valores baixos de RMSF mostram pequenas flutuações e valores altos significam grandes flutuações. Os valores de RMSF observados para os aminoácidos da tríade catalítica Gln19, Cys25 e His162, nas formas holo/apo, foram 0,059/0,049, 0,0411/0,047 e 0,035/0,044 nm, respectivamente. Ademais, exceto para a Gln19, todos os valores de RMSF observados foram menores para a forma holo. De modo geral, os baixos valores de RMSF observados mostram que estes aminoácidos apresentam pequenas flutuações em ambas formas da enzima, ou seja, suas posições são conservadas ao longo do tempo de simulação, dado este muito importante em sistemas biológicos, uma vez que seria esperado que o sítio catalítico tivesse estabilidade estrutural. Observa-se na Figura 7.5 que a região compreendida pelos aminoácidos 56 a 63 apresenta grandes flutuações para ambas formas da enzima. Essa região faz parte do loop da enzima constituída pelos aminoácidos 56-68, e, portanto, esta alta flexibilidade se deve à presença deste loop. Entretanto, para a forma holo, observa-se uma pequena estabilização desse loop, com baixos valores de RMSF. Outra região de alta flexibilidade é a constituída pelos resíduos 93 a 108, que são parte do loop 84-109. Os baixos valores de RMSF para a forma holo nessa região, em comparação com a forma apo, indicam maior estabilização desse loop. Embora essas regiões sejam flexíveis, não afetam a posição da tríade catalítica, uma vez que foram observados baixos valores de RMSF para esses aminoácidos. 


\subsubsection{Ligações de hidrogênio}

Em sistemas biológicos, interações do tipo ligação de hidrogênio são de grande importância para a estabilização de ligantes, cofatores e fármacos em enzimas. Assim, a Figura 7.6 a seguir mostra as ligações de hidrogênio formadas pela cadeia peptídica com a enzima cruzaína. Ligações de hidrogênio foram definidas pela distância máxima entre doador e aceptor de $0,35 \mathrm{~nm}$ e ângulo formado por doador, hidrogênio e aceptor maior que $120^{\circ}$.

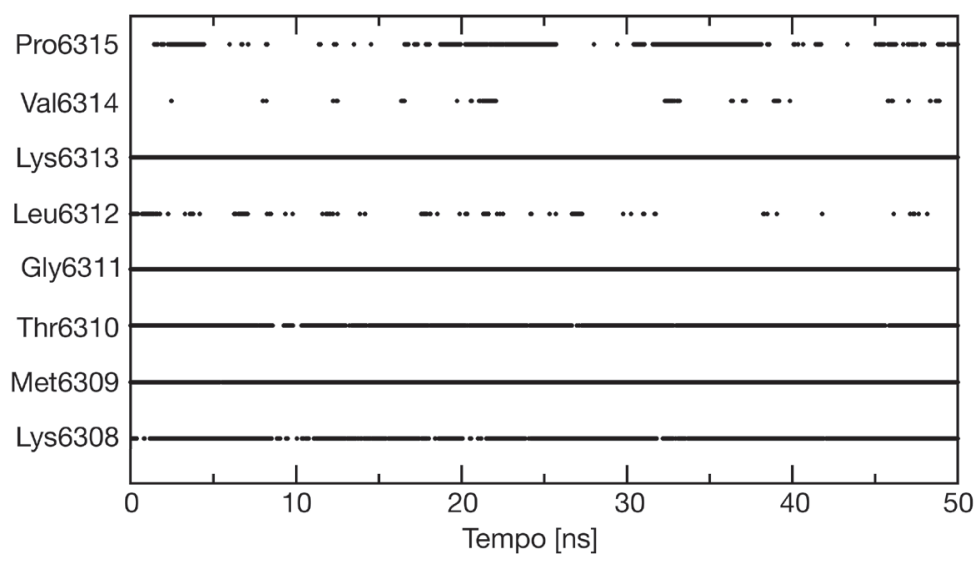

Figura 7.6 Ligações de hidrogênio entre a cadeia peptídica e enzima cruzaína ao longo do tempo de simulação.

Por ser um sistema dinâmico, observar-se na Figura 7.6 a formação e quebra de ligações de hidrogênio ao longo de 50 ns de simulação. Linhas contínuas na Figura 7.6 significam que ocorrem ligações de hidrogênio estáveis ao longo da trajetória. Apesar de a glicina não possuir cadeia lateral com grupos doadores e aceptores de elétron, observa-se a formação de ligação de hidrogênio estável via átomos da ligação peptídica deste com a cruzaína. O mesmo ocorre para a valina, mas, nesse caso, as ligações de hidrogênio formadas não são estáveis ao longo do tempo. A tríade catalítica é rodeada pelos aminoácidos Met6309, Thr6310 e Gly6311 da cadeia peptídica. Portanto, esses aminoácidos contribuem para a estabilização da região da tríade via ligações de hidrogênio estáveis com a enzima cruzaína, conforme Figura 7.4. Em detalhes, o oxigênio carbonílico do resíduo Met6309 faz ligações de hidrogênio com o hidrogênio da ligação peptídica do Trp184 e com o grupo amina da Gln19; o grupo hidroxila do Thr6310 interage com o íon carboxilato do Asp161; e o hidrogênio da ligação peptídica da Gly6311 interage com o oxigênio carbonílico do Asp161. Assim, podem-se usar essas informações para o desenho racional de fármacos, ou seja, estes deveriam ter grupos carregados positivamente (isto é, $\mathrm{NH}_{3}^{+}$) e com grupos doadores ou aceptores de prótons (isto é, $\mathrm{OH}$ ). 


\subsubsection{Análise do sítio ativo}

A literatura cita os resíduos Cys25 e His162 como os responsáveis pela atividade catalítica da cruzaína. Assim, calculou-se, ao longo da simulação, a distância entre o enxofre da Cys 25 e o nitrogênio da His162 para os sistemas estudados. Para a forma holo, a distância foi de $0,34 \mathrm{~nm}$ (d.p. de $0,02 \mathrm{~nm}$ ) e apo, de 0,36 nm (d.p. de $0,03 \mathrm{~nm}$ ). A pequena distância para a forma holo mostra que, como discutido anteriormente, a cadeia peptídica estabiliza a posição dos resíduos catalíticos. De acordo com o mecanismo enzimático proposto para as cisteíno-proteases, a etapa inicial é a transferência do próton (hidrogênio) da Cys25 para a His162. Portanto, a distância de $0,34 \mathrm{~nm}$ é o suficiente para que ocorra esse processo de transferência. Na segunda etapa, o $\mathrm{S}^{1-}$ da Cys 25 ataca covalentemente o carbono carbonílico da ligação peptídica, formando um intermediário tetraédrico, sendo esse oxiânion estabilizado pelo resíduo Gln19. Posteriormente, o fragmento do N-terminal do peptídeo é liberado e a His 162 volta à sua forma desprotonada. Ao longo dessa etapa, ocorre a formação do intermediário tioéster, que liga o novo C-terminal do peptídeo ao tiol da Cys25. Em seguida ocorre a hidrólise da ligação do tioéster, liberando o C-terminal do peptídeo e regenerando a enzima livre. A Figura 7.7A mostra, de forma sucinta, o mecanismo proposto na literatura. Com base nesse mecanismo, ao longo da simulação, foi possível observar vários snapshots com conformação que descrevem muito bem as etapas acima, o que é mostrado na Figura 7.7B.

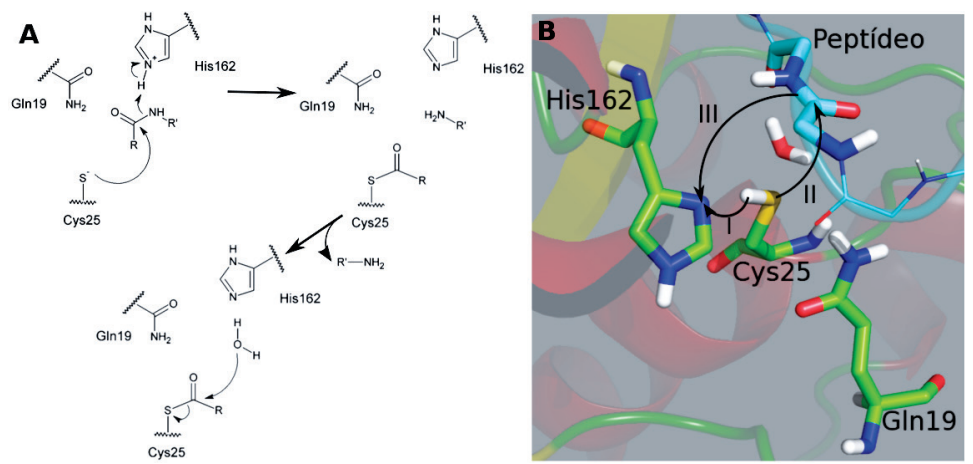

Figura 7.7 (A) Mecanismo enzimático de cisteíno protease proposto na literatura; e (B) snapshot obtido a $50 \mathrm{~ns}$ de simulação por dinâmica molecular.

$\mathrm{Na}$ figura acima, é interessante observar o quanto o arranjo estrutural obtido pela dinâmica molecular (Figura 7.7B) é similar ao proposto na literatura (Figura 7.7A). Na Figura 7.7B observa-se que, no passo I, ocorre a transferência do próton para a histidina e posterior ataque do $\mathrm{S}^{1-}$ no carbono carboxílico do peptídeo (passo II). Posteriormente, no passo III, o N-terminal aceita um próton da histidina e ocorre liberação deste. Paralelamente a esse processo, ocorre a hidrólise da água, que seria o passo IV, o qual não é mostrado na Figura 7.7B. Entretanto, é interessante 
observar que há a presença de uma molécula de água próxima ao carbono carboxílico do peptídeo. Portanto, esse arranjo estrutural pode ser utilizado em cálculos de mecânica quântica para a caracterização do mecanismo enzimático da enzima cruzaína. Para a presente enzima, o mecanismo já é bem conhecido e caracterizado na literatura. Entretanto, para um mecanismo desconhecido ou parcialmente conhecido, a dinâmica molecular se torna uma eficiente técnica para obter amostragem de possíveis arranjos estruturais que podem representar o mecanismo enzimático e posterior caracterização desses arranjos via cálculos de química quântica.

\subsubsection{Geração de ensemble docking}

Uma das principais dificuldades no desenvolvimento racional de fármacos pode ser atribuída à falta de informações no nível atomístico do alvo molecular sob efeito de solvente, temperatura e pressão. Por exemplo, nos cálculos de docking e virtual screening, a enzima é considerada rígida, e o efeito do solvente é desprezado. Nos cálculos mais elaborados, usando as técnicas acima, apenas alguns aminoácidos são considerados flexíveis, e o solvente é representado por uma constante dielétrica. Entretanto, sabe-se que a flexibilidade das enzimas e o efeito do solvente são de extrema importância em sistemas biológicos. Uma metodologia que tem sido utilizada na literatura para considerar o efeito do solvente e a flexibilidade da enzima de interesse é o uso do chamado ensemble docking, ${ }^{30}$ cujo método consiste em utilizar um conjunto de estruturas que podem ser obtidas via ressonância magnética nuclear ou por cálculos teóricos (dinâmica molecular ou Monte Carlo). Posteriormente, podem ser utilizadas na busca sistemática de candidatos a fármacos, via cálculos de docking e virtual screening. Embora o uso de ensemble docking seja interessante e promissor, ainda não existe um protocolo de elaboração dele, sendo utilizado aqui um conjunto de estruturas obtidas a partir da análise de clusters (agrupamento de estruturas semelhantes) da trajetória da dinâmica molecular. A geração de clusters pode ser obtida pela sobreposição de conformações ao longo da simulação e ser usada como critério uma pequena variação entre essas estruturas. Assim, quanto maior for o número de conformações em um cluster, maior será a contribuição da conformação média desse cluster na trajetória da DM. Por ter maior representatividade, esse cluster poderá ser utilizado em cálculos de docking e virtual screening, bem como na construção de modelos farmacológicos. A Figura 7.8 a seguir mostra os ensemble docking obtidos para as formas apo e holo. 

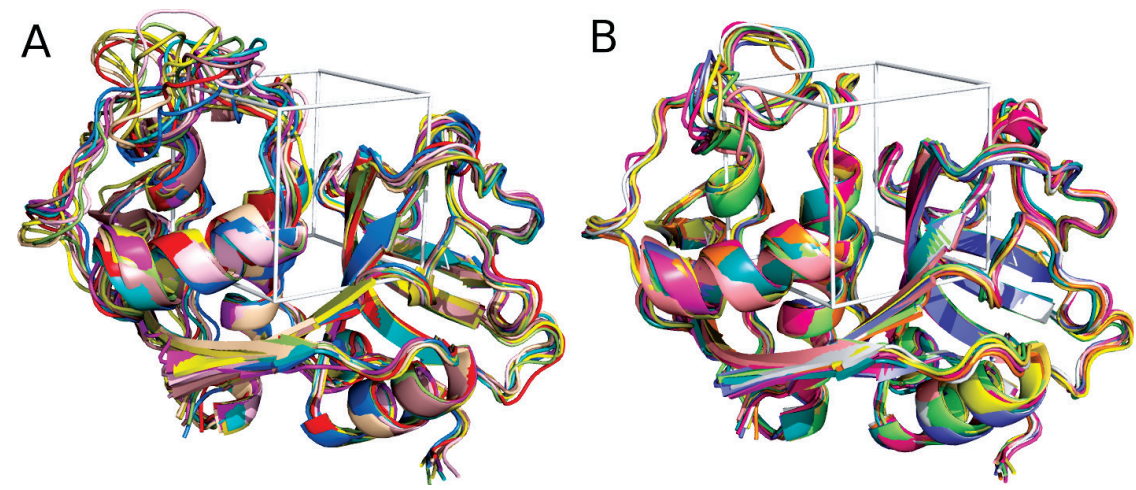

Figura 7.8 Ensembles docking para as formas apo (A) e holo (B) gerados a partir da trajetória de dinâmica molecular. A região do sítio ativo está localizada dentro da caixa branca.

Para a geração dos conjuntos da Figura 7.8, foram considerados os dez maiores clusters obtidos na DM para as formas apo e holo. A Figura 7.8 mostra também a região que poderá ser utilizada para cálculos de docking e virtual screening. Interessante observar que a enzima na forma apo é muito mais flexível que na forma holo, dados estes de acordo com os valores de RMSD (Figura 7.4) e RMSF (Figura 7.5). A partir da elaboração dos conjuntos, estes poderão ser utilizados na busca racional de fármacos via técnicas de docking e virtual screening, bem com na elaboração de modelos farmacológicos (3D-QSAR). A escolha do melhor conjuntos acima poderá ser feita com base nos valores de energia livre de ligação, a partir das técnicas mencionadas, de diferentes compostos complexados com a enzima que corroboram com dados experimentais.

\subsection{Considerações FINAIS}

Dentre as diversas aplicações da técnica de dinâmica molecular, esta tem mostrado papel fundamental no estudo de sistemas biológicos. A partir de dados estruturais e energéticos, que são influenciados pelo efeito de solvente e temperatura, estas aplicações possibilitam o entendimento das interações ligante-receptor. Com este conhecimento, novos candidatos a fármacos podem ser otimizados via inserção de grupos moleculares específicos. Já a combinação da metodologia DM com outras (docking, virtual screening e química quântica) possibilita o desenvolvimento racional de novos candidatos inibidores de alvos de interesse, bem como permite identificar e caracterizar possíveis mecanismos enzimáticos. 


\section{REFERÊNCIAS}

Allen, M. P.; Tildesley, D. J. Computer simulation of liquids. Nova York: Oxford University Press, 1987.

Andricopulo, A. D.; Yunes, R. A.; Nunes, R. J.; Savi, A. O. S.; Cruz, A. B.; Cechinel, V. Synthesis and antibacterial activity of cyclic imides: 3,4 dichloromaleimides and 3-chloro-4-substituted-maleimides. Quím. Nova, 21, p. 573-577, 1998.

Berendsen, H. J. C.; Postma, J. P. M.; Dinola, A.; HaAk, J. R. Molecular dynamics with coupling to an external bath. J. Chem. Phys., 81, 3684, 1984.

Berendsen, H. J. C.; Postma, J. P. M.; VAn Gunsteren, W. F. Interaction models for water in relation to protein hydration. In: Pullman, B. (Ed.). Intermolecular forces: the Jerusalem symposia on quantum chemistry and biochemistry. Dordrecht: Reidel, 1981. p. 331-342.

Berendsen, H. J. C.; Van der Spoel, D.; Van Drunen, R. Gromacs: a message passing parallel molecular dynamics implementation. Comput. Phys. Commun., 91, p. 43-56, 1995.

Cazzulo, J. J.; StokA, V.; Turk, V. Cruzipain, the major cysteine proteinase from the protozoan parasite Trypanosoma cruzi. Bio. Chem., 378, p. 1-10, 1997.

. The major cysteine proteinase of Trypanosoma cruzi: a valid target for chemotherapy of Chagas disease. Curr. Pharma. Design, 7, p. 1143-1156, 2001.

Chen, Y. T.; Brinen, L. S.; Kerr, I. D.; Hansell, E.; Doyle, P. S. In vitro and in vivo studies of the trypanocidal properties of WRR-483 against trypanosoma cruzi. PLoS Negl. Trop. Dis., v. 4, n. 9, e825, 2010.

Coura, J. R.; Castro, S. L. A critical review on Chagas disease chemotherapy. Memb. Inst. Oswaldo Cruz, 97, p. 3-24, 2002.

Craig, I. R.; Essex, J. W.; SpIEgel, K. Ensemble docking into multiple crystallographically derived protein structures: an evaluation based on the statistical analysis of enrichments. J. Chem. Inf. Model., v. 50, n. 4, p. 511-524, 2010.

Dias, L. C.; Dessoy, M. A.; Silva, J. J. N.; Thiemann, O. H.; Oliva, G.; Andricopulo, A. D. Quimioterapia da doença de Chagas: estado da arte e perspectivas no desenvolvimento de novos fármacos. Quím. Nova, v. 32, n. 9, p. 2444-2457, 2009.

Docampo, R. Recent developments in the chemotherapy of Chagas' disease. Curr. Pharm. Des., 7, p. 1157-1164, 2001.

Durrant, J. D.; Keranen, H.; Wilson, B. A.; McCammon, J. A. Computational identification of uncharacterized cruzain binding sites. PLoS Negl. Trop. Dis., v. 4, n. 5, 2010.

Fairlamb, A. H. Future prospects for the chemotherapy of Chagas' disease. Medicina, Buenos Aires, 59, p. 179-187, 1999.

Jorgensen, W. L.; Maxwell, D. S.; Tirado-Rives, J. Development and testing of the OPLS all-atom force field on conformational energetics and properties of organic liquids. J. Am. Chem. Soc., v. 118, n. 45, p. 11225-11236, 1996. 
Leung, D.; Abbenante, G.; Fairlie, D. P. Protease inhibitors: current status and future prospects. J. Med. Chem., 43, n. 3, p. 305-341, 2000.

Lindahl, L.; Hess, B.; VAND der Spoel, D. Gromacs 3.0: a package for molecular simulation, and trajectory analysis. J. Mol., v. 7, n. 8, p. 306-317, 2001.

Malvezzi, A.; Rezende, L.; Amaral, A. T. Pharmacophore model of cruzain inhibitors. QSAR Comb. Sci., 28, n. 8, p. 781-784, 2009.

McKerrow, J. H.; Caffrey, C.; Kelly, B.; Loke, P.; Sajid, M. Proteases in parasitic diseases. Annual Review of Pathology: mechanisms of disease, 1, p. 497-536, 2006.

McKerrow, J. H.; Rosenthal, P. J.; Swenerton, R.; Doyle, P. Development of protease inhibitors for protozoan infections. Curr. Opin. Infc. Dis., 21, p. 668-672, 2008.

Mladenovic, M.; Ansorg, K.; Fink, R. F.; Thiel, W.; Schirmeister, T.; Engels, B. Atomistic insights into the inhibition of cysteine proteases: first QM/MM calculations clarifying the stereoselectivity of epoxide-based inhibitors. J. Phys. Chem. B, 112, 11798-11808, 2008.

Moncayo, A. Chagas disease: current epidemiological trends after the interruption of vectorial and transfusional trasmission in the Southern Cone countries. Mem. Inst. Oswaldo Cruz, 98, p. 577-591, 2003.

Moncayo, A.; Ortiz Yanine, M. I. An update on Chagas disease (human American trypanosomiasis). Ann. Trop. Med. Parasitol., 100, p. 663-677, 2006.

Oliveira, O. V.; Santos, J. D.; Freitas, L. C. G. Molecular dynamics simulation of the gGAPDH-NAD ${ }^{+}$complex from Trypanosoma cruzi. Molecular Simulation, 38, 13, p. 1124-1131, 2012.

Rawlings, N. D.; Barrett, A. J.; Bateman, A. Merops: the peptidase database. Nucleic Acids Res., 38, p. D227-D233, 2010.

Reis, F. C.; Judice, W. A.; Juliano, M. A.; Juliano, L.; Scharfstein, J.; Lima, A. P. The substrate specificity of cruzipain 2, a cysteine protease isoform from Trypanosoma cruzi. FEMS Microbiology Letters, 259, p. 215-220, 2006.

Rodriguez, J. B. Specific molecular targets to control tropical diseases. Curr. Pharm. Des., 7, 1105-1116, 2001.

Romeiro, N. C.; Aguirre, G.; Hernández, P.; González, M.; Cerecetto, H.; Aldana, I.; Pérez-Silanes, S.; Monge, A.; Barreiro, L. J.; Lima, L. M. Synthesis, trypanocidal activity and docking studies of novel quinoxaline-N-acylhydrazones, designed as cruzain inhibitors candidates. Bioorg. \& Med. Chem., 17, p. 641-652, 2009.

Rueda, M.; Bottegoni, G.; Abagyan, R. Recipes for the selection of experimental protein conformations for virtual screening. J. Chem. Inf. Model., 50, n. 1, p. 186-193, 2010.

Salmon, L.; Landry, V.; Melnyk, O.; Maes, L.; Shergheraert, C.; Davioud-Charvet, E. A. A general approach to the synthesis of polyamine linked-monoindolylmaleimides, a new series of trypanothione reductase inhibitors. Chem. Pharm. Bull., v. 46, n. 707, 1998. 
Schofield, C. J.; Jannin, J.; Salvatella, R. The future of Chagas disease control. Trends Parasitol., 22, p. 583-588, 2006.

Soeiro, M. N. C.; Castro, S. L. Trypanosoma cruzi targets for new chemotherapeutic approaches. Expert Opin. Therap. Targets, 13, p. 105-121, 2009.

Souza, D. H. F.; Garratt, R. C.; Araujo, A. P. U.; Guimarães, B. G.; Jesus, W. D. P.; MiChaels, P. A. M.; Hannaert, V.; Oliva, G. Trypanosoma cruzi glycosomal glyceraldehyde-3-phosphate dehydrogenase: structure, catalytic mechanism and targeted inhibitor design. FEBS Letters, 242, p. 131-135, 1998.

Turk, B. Targeting proteases: successes, failures and future prospects. Nature Reviews Drug Discovery, 5, p. 785-799, 2006.

Urbina, J. A.; Docampo, R. Specific chemotherapy of Chagas disease: controversies and advances. Trends Parasitol., v. 19, n. 11, p. 495-501, 2003.

World Health Organization (WHO). Control of Chagas disease: second report of the WHO expert committee. Genebra: WHO, 2002. (Technical Report Series, 905).

Zanatta, N.; Amaral, S. S.; Santos, J. M.; Mello, D. L.; Fernandes, L. S.; Bonacorso, H. G.; Martins, M. A. P.; Andricopulo, A. D.; BorchHardT, D. M. Convergent synthesis and cruzain inhibitory activity of novel 2-(N\$-benzylidenehydrazino)-4-trifluoromethylpyrimidines. Biorg. Med. Chem., 16, p. 10236-10243, 2008.

Zuccotto, F.; Brum, R.; Pacanowska, D. G.; Perez, L. M. R.; Gilbert, I. H. The structure-based design and synthesis of selective inhibitors of Trypanosoma cruzi dihydrofolate reductase. Bioorg. Med. Chem. Lett., 9, p. 1463-1468, 1999. 
ESTE LIVRO FOI IMPRESSO EM JULHO DE 2018 PELA PSI7 Printing Solutions \& Internet 7 S.A. em São Paulo/SP. 